\title{
Scaling carbon sequestration phenomena to the earth's surface
}

\author{
Saumik Dana ${ }^{a}$ \\ ${ }^{a}$ University of Southern California, Los Angeles, 90007, CA, USA
}

\begin{abstract}
We present a computational framework for fast monitoring of fault stability and ground deformation in multiphase geomechanics and demonstrate its efficacy for a carbon sequestration-enhanced oil recovery case study. The staggered solution algorithm for the coupled problem is augmented with a feature that allows for the flow and geomechanics sub-problems to be solved on different unstructured tetrahedral grids. For the field scale problem, the geomechanics grid goes all the way to the free surface while the flow grid is truncated at a depth above which the layers are impermeable. This framework avoids the unnecessary computational burden associated with equilibrating the initial pressure solution in the overburden, allows for a study of the critical interaction between overburden and faults, allows for fast renditions of ground deformation, and allows a choice of resolution for the flow and geomechanics grids independently to capture disparate length scales of the underlying physics.
\end{abstract}

Keywords: Carbon sequestration, Multiphase geomechanics, Fault stability, Ground deformation, Unstructured tetrahedral grids, Computational geometry

\section{Introduction}

The US Department of Energy Regional Carbon Sequestration Partnership (RCSP) Initiative (see Fig. 1) was launched in year 2003 to advance development and testing of effective carbon capture, utilization and storage (CCUS) technologies across the United States. With the amount of planned $\mathrm{CO}_{2}$ injection in these projects in the order of millions of metric tonnes, the quantification of geomechanical impact becomes critical in order to create guiding protocols for the design and monitoring of CCUS operations [3-14]. 


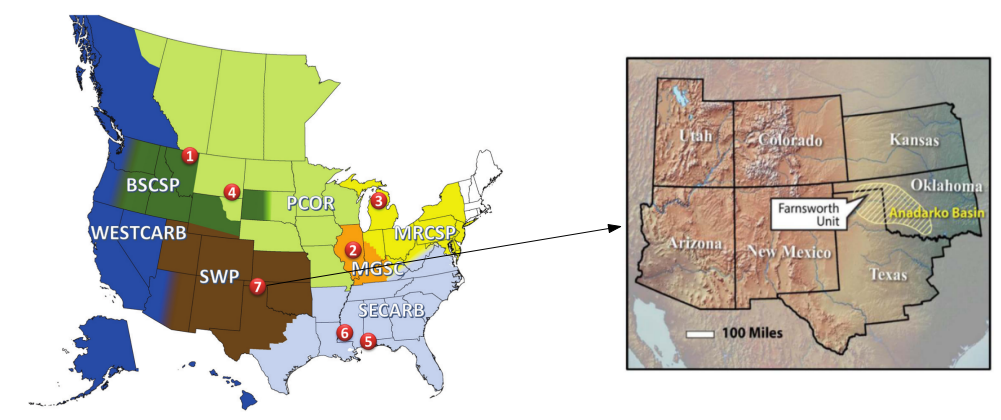

Figure 1: Locations of RCSP large-scale projects (Source: [1]) and geologic extent of Farnsworth (Source: [2])

Given the issues of poor resolution, interpretation uncertainty and partial coverage that are associated with geodetic (GPS and InSAR) measurements and recent push towards continuous monitoring of injection sites as part of the traffic light system [15], the need for predicting ground deformation to monitor subsidence or uplift has grown. Moreover, this capability is critical in cases where multiple forward simulation runs are performed as part of an inverse analysis to estimate the subsurface porosity/permeability fields from ground deformation data obtained from InSAR and/or GPS. Typically, the numerical grid in the region where the bulk of the flow-geomechanical coupling activity takes places carries a resolution that is required for the simulation accuracy. That region is typically the thin sliver that represents the reservoir. To simulate ground deformation, and any local geomechanical activity in the overburden would require a mesh that is extruded from the reservoir grid and extended all the way to the ground surface. This makes the geomechanical grid computationally expensive.

As a result, it is optimal to truncate the flow grid to a certain depth above which the flow activity is not the subject of interest. But the problem is that in case of the single grid approach, since both flow and geomechanics are resolved on the same grid, even the geomechanical grid would need to be truncated at the same depth, thereby obviating a study of ground deformation, and indeed any local geomechanical activity in the overburden. As we shall see in the field case, the overburden carries a layered structured with different rock lithologies, and any study that truncates the geomechanical grid at the reservoir depth would not allow encompassing geomechanical processes, e.g. caprock fracturing, above the reservoir for the concomitant study from a geomechanical standpoint. 


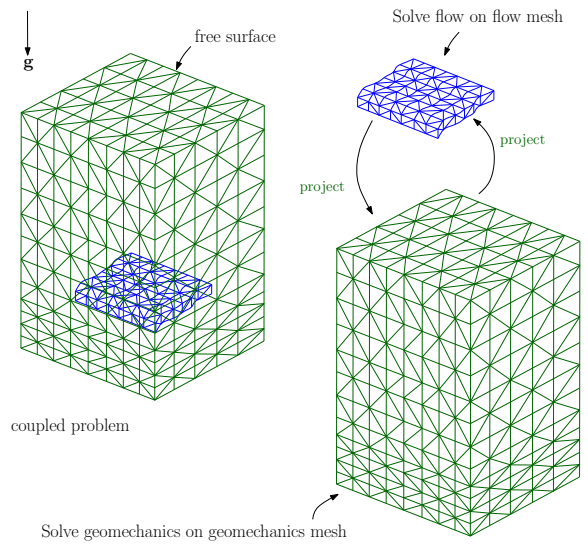

(a) Enabling simultaneous study of fault stability and ground deformation

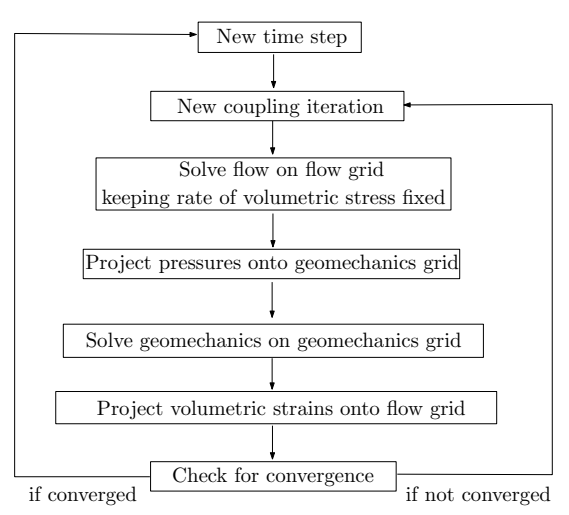

(b) Flowchart

Figure 2: The two grid method

In lieu of all of the above, we present a technique which allows for spatial decoupling of the flow and geomechanics domains with the subproblems being resolved on separate grids (Fig. 2a). The geomechanical equations are solved using the finite element method whereas the multiphase flow equations are solved using the finite volume method. Typically, in such problems, the geomechanics mesh is expected to be coarser than, or the same, as the flow mesh everywhere the two meshes co-exist, but we generalize the method to the cases where the geomechanics elements can be smaller than the flow elements in localized regions where capturing the mechanics is more pertinent, e.g. a basement fault below the reservoir, a bounding fault outside the reservoir, or fractures in the caprock, all of which may slip due to stresses transferred from the reservoir. Furthermore, we would like to generalize the notion of the two-grid from structured hexahedral meshes [16] to unstructured tetrahedral meshes. As shown in Fig. 2b, the two-grid approach is built on top of the popular fixed stress split iterative scheme [16-52].

\section{Governing equations}

Under the quasi-static assumption for earth displacements, the governing equation for linear momentum balance of the solid/fluid system can be expressed as

$$
\nabla \cdot \boldsymbol{\sigma}+\rho_{b} \boldsymbol{g}=\mathbf{0}
$$


where $\boldsymbol{\sigma}$ is the Cauchy total stress tensor, $\boldsymbol{g}$ is the gravity vector, and $\rho_{b}=$ $\phi \sum_{\beta}^{n_{\text {phase }}} \rho_{\beta} S_{\beta}+(1-\phi) \rho_{s}$ is the bulk density, $\rho_{\beta}$ and $S_{\beta}$ are the density and saturation of fluid phase $\beta, \rho_{s}$ is the density of the solid phase, $\phi$ is the true (Eulerian) porosity, and $n_{\text {phase }}$ is the number of fluid phases. Note that, by definition, the sum of all fluid phase saturations satisfies $\sum_{\beta}^{n_{\text {phase }}} S_{\beta} \equiv 1$. Assuming that the fluids are immiscible, the mass-conservation equation for each phase $\alpha$ is

$$
\frac{d m_{\alpha}}{d t}+\nabla \cdot \boldsymbol{w}_{\alpha}=\rho_{\alpha} f_{\alpha},
$$

where the accumulation term $d m_{\alpha} / d t$ describes the time variation of fluid mass relative to the motion of the solid skeleton, $\boldsymbol{w}_{\alpha}$ is the mass-flux of fluid phase $\alpha$ relative to the solid skeleton, and $f_{\alpha}$ is the volumetric source term for phase $\alpha$ that can model the effect of wells. Eqs. (1) and (2) are coupled by virtue of poromechanics. Following [53], the poroelasticity equations can be written in incremental form as

$$
\delta \boldsymbol{\sigma}=\boldsymbol{C}_{d r}: \delta \boldsymbol{\varepsilon}-b \delta \tilde{p} \mathbf{1} \equiv \boldsymbol{C}_{d r}: \delta \varepsilon-\sum_{\beta} b_{\beta} p_{\beta} \mathbf{1}
$$

where $\tilde{p}=\sum_{\beta} S_{\beta} p_{\beta}$, and $b_{\beta}=b S_{\beta}$ are the Biot coefficients for individual phases such that $\sum_{\beta} b_{\beta}=b, \boldsymbol{C}_{d r}$ is the rank-4 drained elasticity tensor, $\mathbf{1}$ is the rank-2 identity tensor, $b$ is the Biot coefficient of the saturated porous material, $\boldsymbol{\varepsilon}$ is the linearized strain tensor, defined as the symmetric gradient of the displacement vector $\boldsymbol{u}$,

$$
\boldsymbol{\varepsilon}:=\frac{1}{2}\left(\nabla \boldsymbol{u}+\nabla^{\mathrm{T}} \boldsymbol{u}\right) \equiv \frac{1}{3} \varepsilon_{v} \mathbf{1}+\boldsymbol{e},
$$

where $\varepsilon_{v}=\operatorname{tr}(\varepsilon)$ is the volumetric strain and $\boldsymbol{e}$ is the deviatoric strain tensor. In the deformed configuration, the mass of phase $\alpha$ per unit volume of porous medium is

$$
m_{\alpha}=\rho_{\alpha} S_{\alpha} \phi J
$$

where $J=1+\varepsilon_{v}$ is the Jacobian of deformation under infinitesimal deformation theory. In lieu of Eqs. (3)-(5), Eq. (2) can be rewritten as

$$
\frac{\partial}{\partial t}\left(\rho_{\alpha} \sum_{\beta}\left(N_{\alpha \beta}+\frac{b_{\alpha} b_{\beta}}{K_{d r}}\right) p_{\beta}\right)+\frac{1}{K_{d r}} \frac{\partial}{\partial t}\left(\rho_{\alpha} b_{\alpha} \sigma_{v}\right)+\nabla \cdot \boldsymbol{w}_{\alpha}=\rho_{\alpha} f_{\alpha}
$$

where $N_{\alpha \beta}$ is given in terms of saturation, porosity, fluid compressibility, Biot coefficients, drained modulus and the slope of the capillary pressure curve 
The derivation of Eq. 6 is given in Appendix A. Because we assume that the fluids are immiscible, the mass-flux of phase $\alpha$ is $\boldsymbol{w}_{\alpha}=\rho_{\alpha} \boldsymbol{v}_{\alpha}$, where we adopt the traditional multiphase-flow extension of Darcy's law [54, 55]:

$$
\boldsymbol{v}_{\alpha}=-\frac{\boldsymbol{k} k_{\alpha}^{r}}{\mu_{\alpha}}\left(\nabla p_{\alpha}-\rho_{\alpha} \boldsymbol{g}\right),
$$

where $\mu_{\alpha}$ and $k_{\alpha}^{r}$ are the dynamic viscosity and the relative permeability of phase $\alpha$ in presence of other fluid phases. For single-phase water flow, $\alpha=\beta=w, P_{\alpha \beta}=0, S_{\alpha}=1, c_{\alpha}=c_{w}$, and $k_{\alpha}^{r}=1$, as a result of which Eq. (6) reduces to

$$
\frac{\partial}{\partial t}\left(\rho_{w}\left(\frac{1}{M}+\frac{b^{2}}{K_{d r}}\right) p_{w}\right)+\frac{1}{K_{d r}} \frac{\partial}{\partial t}\left(\rho_{w} b \sigma_{v}\right)+\nabla \cdot\left(\rho_{w} \boldsymbol{v}_{w}\right)=\rho_{w} f_{w} .
$$

where $\frac{1}{M}=\phi c_{w}+\frac{(b-\phi)(1-b)}{K_{d r}}$ is the inverse Biot modulus.

\section{Two-grid algorithm and computational framework}

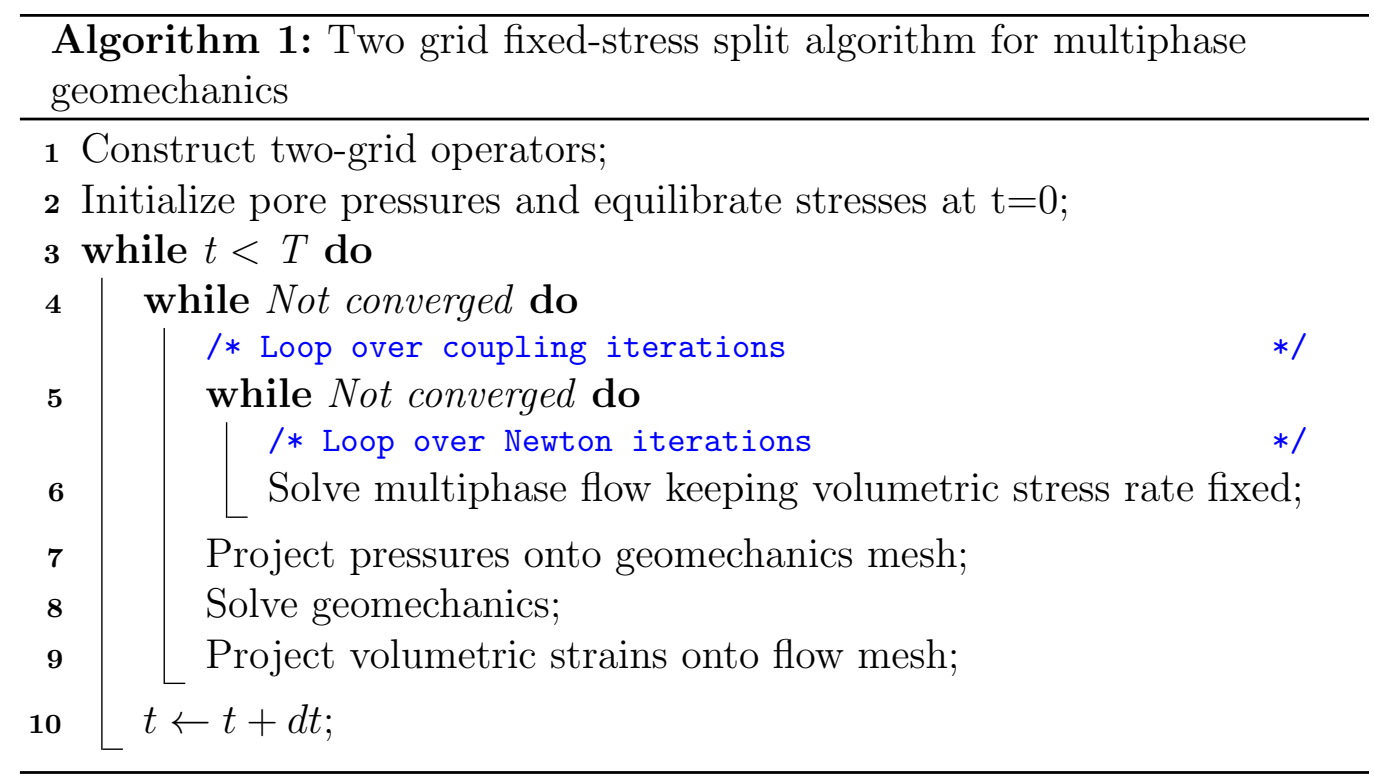

The fully discrete system of equations is presented in Appendix B. As shown in Algorithm 1, the basic idea of the two-grid approach is to transfer pore pressures from the flow mesh to the geomechanics mesh after the flow 


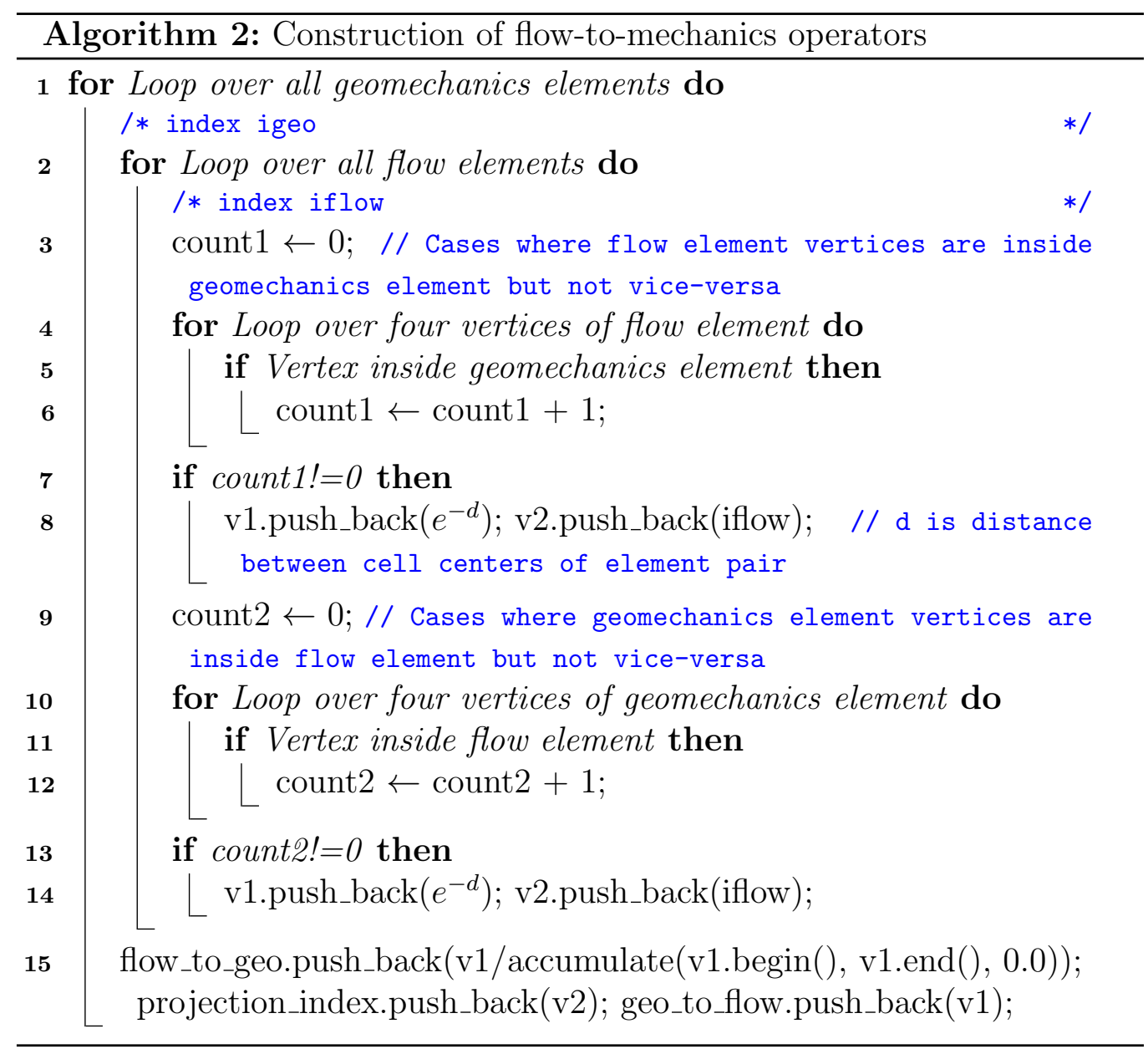

solve and conversely transfer volumetric strain from the geomechanics mesh to the flow mesh after the geomechanics solve in every coupling iteration in every time step. The transferred pore pressure at every geomechanics element is a weighted average of the pore pressures at the collection of flow elements interacting with the geomechanics element. Likewise, the transferred volumetric strain at each flow element is a weighted average of the volumetric strains at the collection of geomechanics elements interacting with the flow element. The weights are the inverse exponential of the distances between the cell centers of the element pair. The reason for choosing the inverse exponential is to factor in the possibility of extremely low values for this distance for element pairs very close to each other. The collection of elements is ob- 

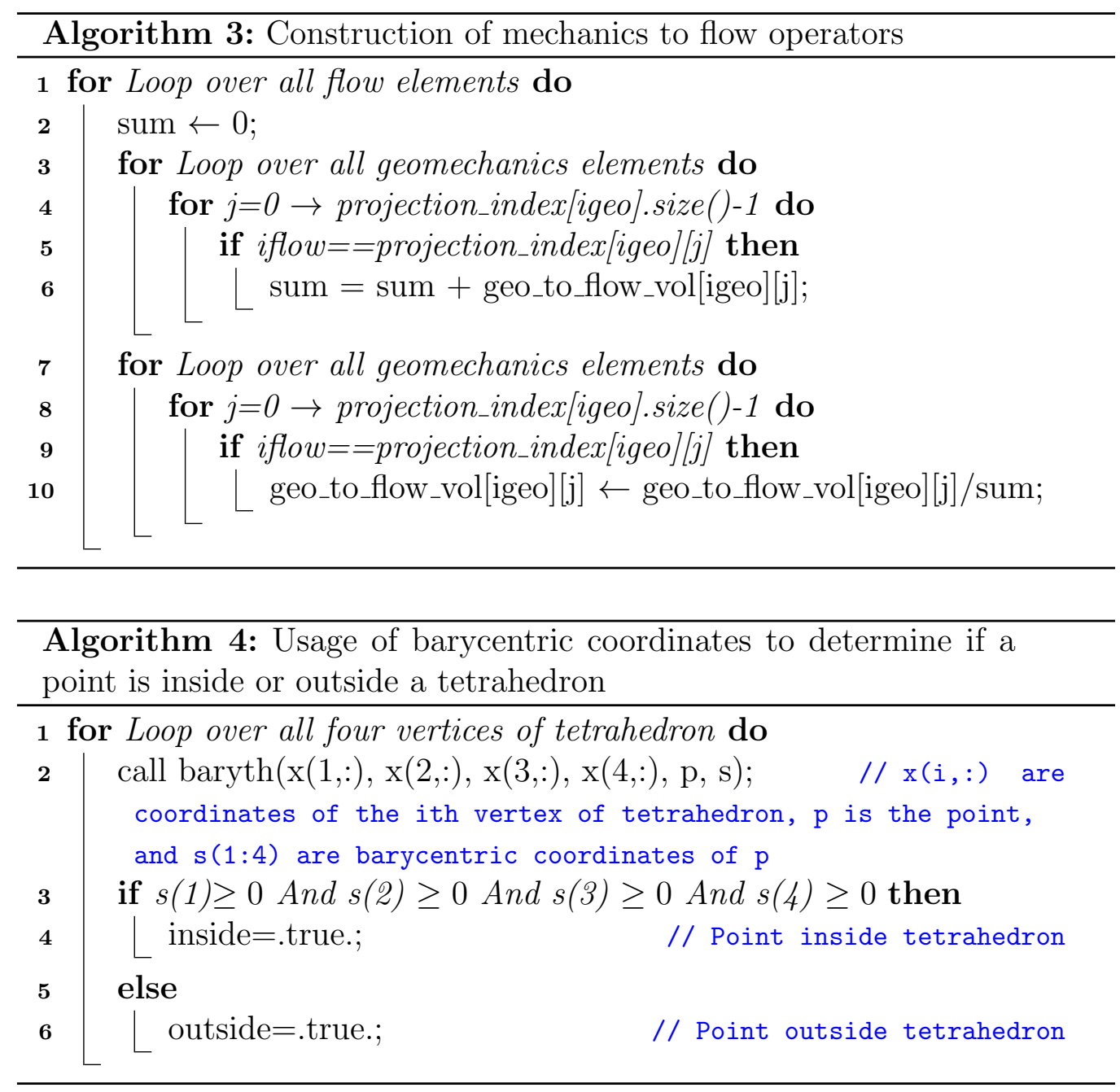

tained by checking all flow elements which have atleast one vertex inside the geomechanics element as well as flow elements which have atleast one vertex of the geomechanics element inside it, as explained in Algorithms 2 and 3. We use barycentric coordinates to determine if a point is inside or outside a tetrahedron, as explained in Algorithm 4. The computation of pressures at a geomechanics element and volumetric strains at a flow elements are elucidated in Figs.3a and 3b respectively. We use STL vectors [56] to deploy the two grid projection operators, as shown in Algorithm 2. 


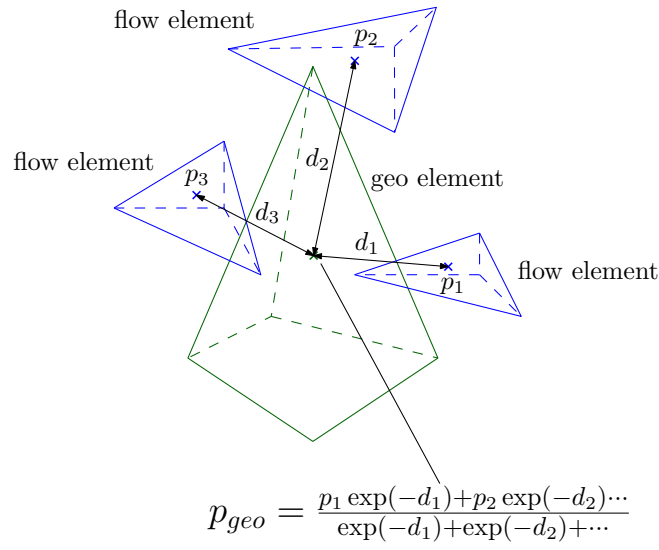

(a) Flow to geomechanics operator

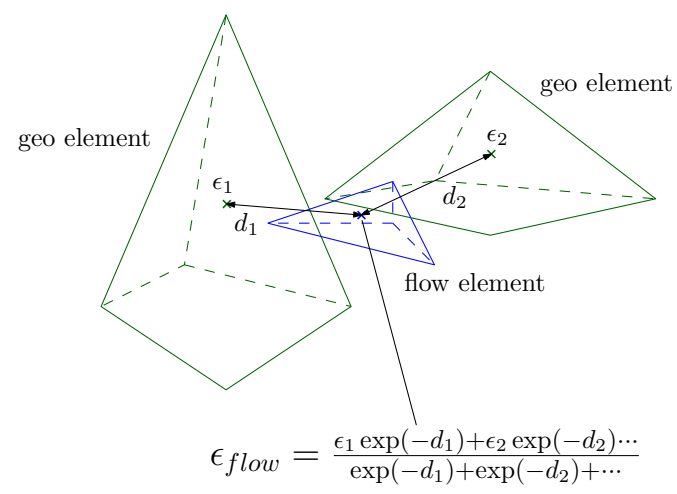

(b) Geomechanics to flow operator

Figure 3: $d$ is a generic variable used to denote the distance between the cell centers of an element pair

\section{Farnsworth Unit field simulation}
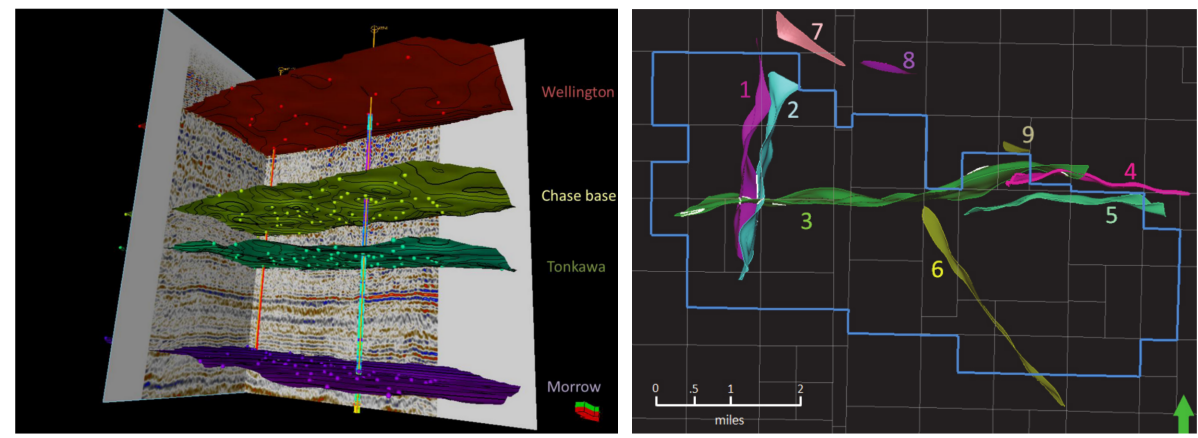

Figure 4: Faults within and near the Farnsworth Unit boundary (blue outline) along the Morrow layer. Source: [57]

The study area for this work is the $\mathrm{CO}_{2}$ storage site called the Farnsworth Unit (FWU) oilfield, a deep structural basin approximately 70,000 square miles in size covering the northern Texas panhandle, western Oklahoma, eastern Colorado and western Kansas (see Fig. 1). As shown in Fig. 4, nine faults which intersected the Morrow-B sandstone reservoir and the Thirteen Finger limestone caprock were identified within FWU during 3-D seismic interpretation [58-62]. Because of their locations, Faults 1 through 3 have the greatest potential for impacting hydrocarbon production and carbon seques- 


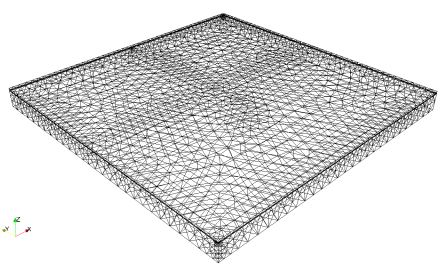

(a) Flow mesh, 31882 elements

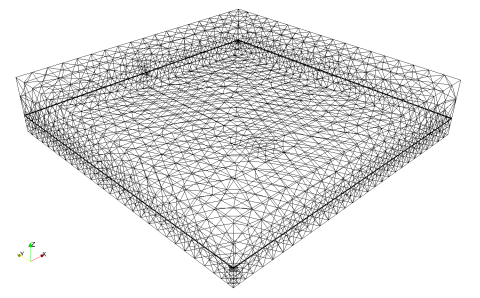

(b) Geomechanics mesh, 42108 elements

Figure 5: Separate flow and geomechanics grids for two-grid method

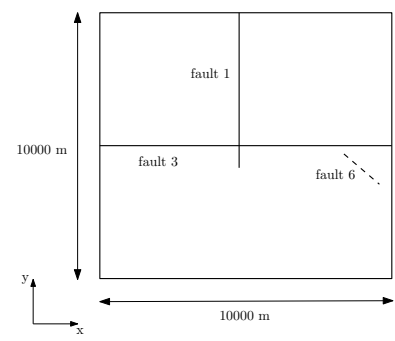

(a) Top view of flow domain, no flow boundary conditions on all boundaries

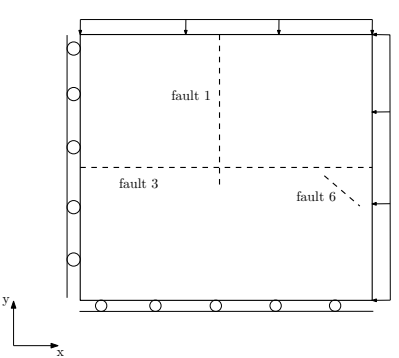

(b) Top view of geomechanics domain

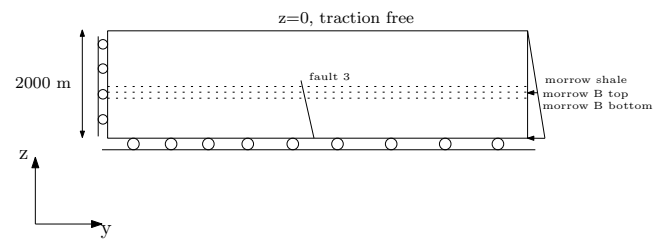

(c) Side view of geomechanics domain

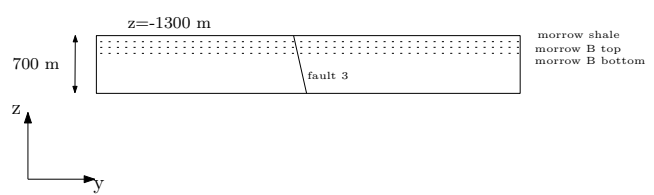

(d) Side view of flow domain

Figure 6: Boundary conditions for the two grid simulation

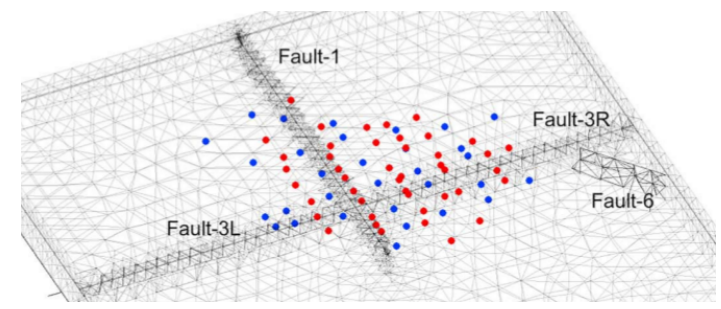

Figure 7: Injectors are shown with blue dots, and producers are shown with red dots

tration at FWU [57]. A conservative estimate of $\mathrm{CO}_{2}$ storage capacity of the injection zone exceeds 10 million tonnes [58-63].

As shown in Fig. 5, we employ two different grids for Flowsim and PyLith 
to use our two-grid method. As shown in Fig. 6, we consider faults 1, 3 and 6 in our calculations. Faults 1 and 3 are cutting through the model while fault 6 ends below the reservoir layer. Fault 3 is located near the center of the model and dips toward south with a $6^{\circ}$ angle. Similarly, Fault 1 is bending to west with an angle of $6^{\circ}$. Both faults 1 and 3 cross through the three horizons. However, Fault 6 is younger, that is, more recent than Morrow-B Sandstone, and terminates just below the reservoir. We assume fault 6 to be vertical based on regional geology. Since Faults 1 and 3 are intersecting with each other, they will share the same grid elements around the intersection region. Thus, for these elements, it is difficult to determine which fault movement to follow during simulations of fault slip. We address this situation for both meshes by splitting Fault 3 into two smaller faults, Fault 3L and 3R, resulting in a gap between Faults 3L and 1 and between Faults $3 \mathrm{R}$ and 1 . It is important to note that while the flow and geomechanics meshes are different, the faults are at the same physical location in both meshes. Transmissibilities of the three faults are set to zero. Compared to the scenario with conductive faults, zero-transmissibility faults cause the reservoir pressure changes resulting from fluid injection and production to be larger. This induces larger changes in the fault stresses, which allows us to simulate a mechanically conservative scenario in the field with regard to the maximum allowable $\mathrm{CO}_{2}$ injection pressure and storage capacity

The simulation spans approximately 57 years of production and injection from January 1956 to June 2013. It is important to keep in mind the field was discovered in 1955 and was unitized in 1963. Secondary recovery commenced in 1964 and $\mathrm{CO}_{2}$ injection began in December 2010. So, although the field is now primarily a carbon storage site, the production/injection history reflects a net fluid withdrawal since it was producing oil since early 1956 to late 2010. As shown in Fig. 7, there are 76 wells in the field: 45 producers and 31 injectors. We use a three phase-three component black-oil model [64] to represent the multiphase flow system during flow simulation. The black-oil flow model must be initialized with phase-dependent fluid properties: compressibility, viscosity, and the dissolved phase volume fraction as functions of the fluid phase pressure. We provide these properties for oil, water, and gas phases that are representative of FWU fluids at reservoir conditions. No-flow boundary condition is imposed on all boundaries of the flow domain. Two of the lateral sides of the geomechanics domain is subject to zero normal displacement boundary condition while the other two lateral sides are subject to traction boundary conditions. The top surface of geomechanics domain, 
which is the ground surface, is traction free while the bottom surface is subject to zero normal displacement boundary condition. The flow domain is truncated at $z=-1300 \mathrm{~m}$. The geomechanics domain goes all the way to the free surface $z=0$. Areal extent of both the domains is $10000 \mathrm{~m} \times 10000$ $\mathrm{m}$. Both the domains extend up to $z=-2000 \mathrm{~m}$. The simulations were performed using the Amazon Web Services instance c5a.8xlarge using 16 MPI processes.

The model is populated with poromechanical properties obtained using two representative well logging data sets. Different reservoir properties are assigned along and across the layers to model an anisotropic response. The average bulk density is $\rho_{b}=2480 \mathrm{~kg} / \mathrm{m}^{3}$, the average sonic compressional velocity is $V_{p}=3600 \mathrm{~m} / \mathrm{s}$, and the average sonic shear velocity is $V_{s}=1920$ $\mathrm{m} / \mathrm{s}$. Dynamic values of the bulk modulus and Young's modulus can be calculated as follows:

$$
K=\rho_{b}\left(V_{p}^{2}-\frac{4}{3} V_{s}^{2}\right), \quad E=\frac{\rho_{b} V_{s}^{2}\left(3 V_{p}^{2}-4 V_{s}^{2}\right)}{V_{p}^{2}-V_{s}^{2}}
$$

The values are $K=19.95 \mathrm{GPa}$ and $E=23.79 \mathrm{GPa}$. The Biot coefficient is assumed to be $b=0.6$, which is representative of consolidated clastic formations in FWU. Reservoir permeability values are obtained by mapping the permeability field of an older simulation model [58-62] on to the coupled geomechanical model. The older model is constructed in Eclipse, is uncoupled to geomechanics, and has been used to track $\mathrm{CO}_{2}$ migration and oil recovery in FWU. Also, the uncoupled Eclipse model covers a smaller area and has smaller mesh elements compared to the coupled geomechanical model. As a result, the coupled model overlaps only partially with the uncoupled model. We average the property value of all the elements of the uncoupled model that are intersected by a coupled model element to obtain the property value of the coupled element. In the non-overlapping region, the geomechanical elements are assigned the field-averaged value of the respective property. We assume a uniform initial porosity of 0.3 in the flow model. This honors the historical production and injection volumes and avoids complexity due to heterogeneity in the porosity field.

\subsection{DCFF time series}

In this study, the FWU faults do not fail, which agrees with observations in the field. Our focus is on quantifying the likelihood of failure or the 


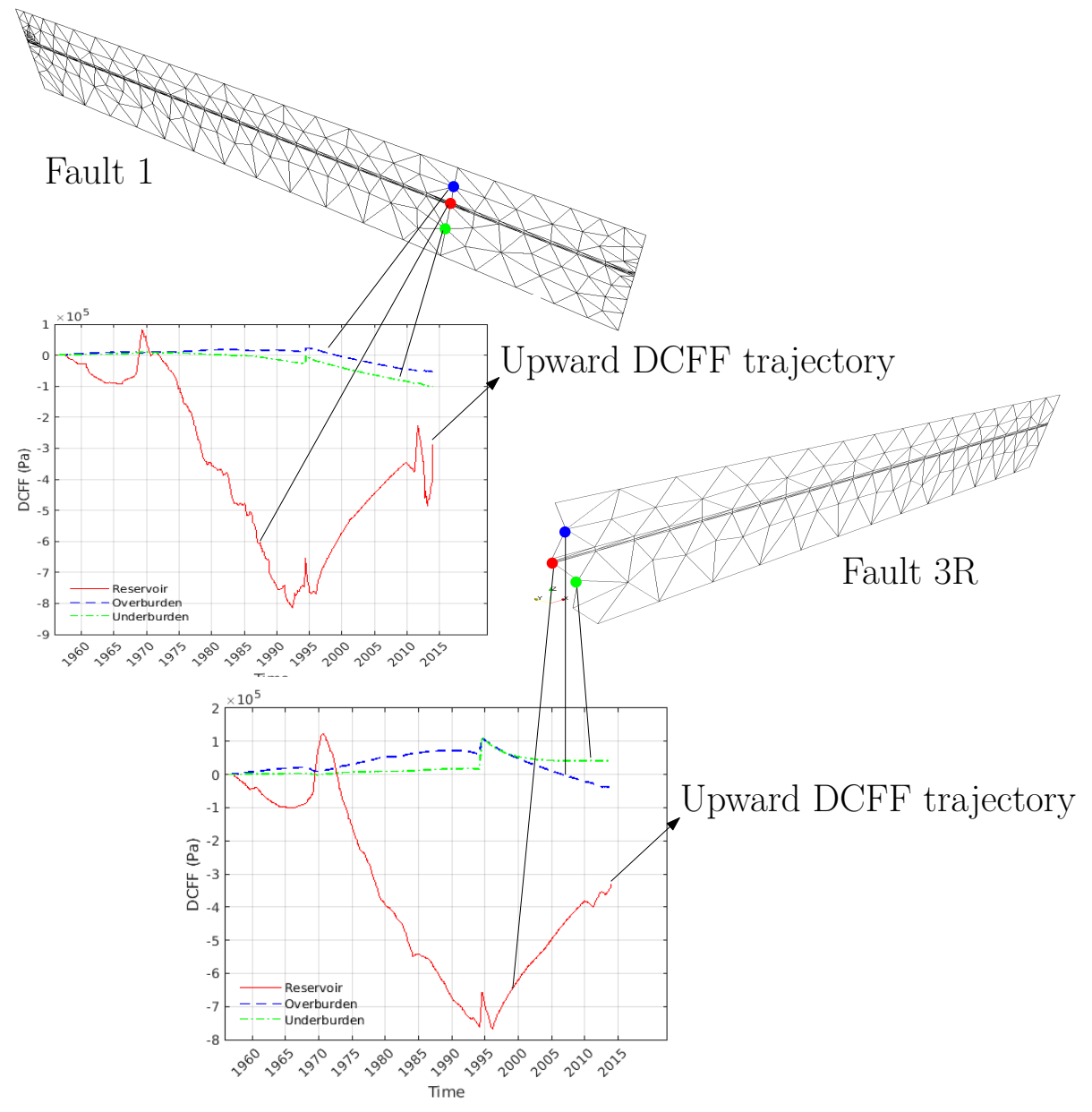

Figure 8: DCFF time series for nodes along the intersection of faults 1 and 3, and for nodes on fault $3 \mathrm{R}$ along the line closest to fault 1

change in mechanical stability induced by production and injection. The likelihood of fault failure is assessed by evaluating the change in Coulomb Failure Function, DCFF, which is a function of the changes in pressure and stress around the fault

$$
\begin{aligned}
\mathrm{DCFF} & =\operatorname{CFF}(t)-\operatorname{CFF}(0) \equiv\left(|\boldsymbol{\tau}|+\mu_{f} \sigma_{n}^{\prime}\right)_{t}-\left(|\boldsymbol{\tau}|+\mu_{f} \sigma_{n}^{\prime}\right)_{0}=\Delta|\boldsymbol{\tau}|+\mu_{f} \Delta \sigma_{n}^{\prime} \\
& \equiv \Delta|\boldsymbol{\tau}|+\mu_{f} \overbrace{\left(\Delta \sigma_{n}+b \Delta p_{f}\right)}^{\Delta \sigma_{n}^{\prime}}
\end{aligned}
$$


where $\Delta|\boldsymbol{\tau}|$ is the magnitude of change in the shear traction vector, $\mu_{f}$ is the fault friction coefficient, $\Delta \sigma_{n}$ is the change in total normal traction and $\Delta p_{f}$ is the change in fault pressure. Here, we consider tension to be positive and compression to be negative, so a positive DCFF indicates a destabilizing effect on the fault. We assume a uniform static friction coefficient value of $\mu_{f}=0.6$ on all the faults.

It is evident from Fig. 8 that the DCFF time series for nodes inside the reservoir closely follow the trends of the time series for average reservoir pressure. When the average reservoir pressure drops, the DCFF reduces whereas when the average reservoir pressure increases, the DCFF increases. This implies that the fault is stable along the reservoir when there is net fluid withdrawal and tends to be unstable when there is net fluid injection. This also follows the trend that is projected by Eq. (9) which states that the DCFF increases when the fault pressure increases and reduces when the fault pressure reduces. We also note that as the $\mathrm{CO}_{2}$ storage is proceeding, the DCFF at the reservoir nodes is continuing to follow a monotonically increasing trend. We also observe that the fault nodes at the overburden and underburden, the trend is that the DCFF increases when the average reservoir pressure decreases and decreases when the average reservoir pressure increases. This suggests that fault is stable along the overburden and underburden when there is net fluid injection and tends to be unstable along the overburden and underburden when there is net fluid withdrawal. The established hypothesis in [3] that fluid injection destabilizes a fault located in the region where the pore pressure has increased, whereas fluid withdrawal destabilizes a fault at the region generally outside the reservoir, lends credence to this observation.

\subsection{Ground deformation}

As shown in Fig. 9, the free surface experiences subsidence, with more subsidence observed at regions where the producer wells are present. From the elasticity theory, the ground deformation pattern is expected to show a smoother version of the subsurface deformation pattern, which results from superposition of deformation fields of individual well activities. This demonstrates the power of our two-grid framework; it allows building models that extend up to the ground surface and predict horizontal and vertical ground deformation, which can be compared against geodetic (GPS and InSAR) measurements. Such comparisons are useful for calibration of coupled models, assimilation of multiphysics data, and estimation of uncertain rock 


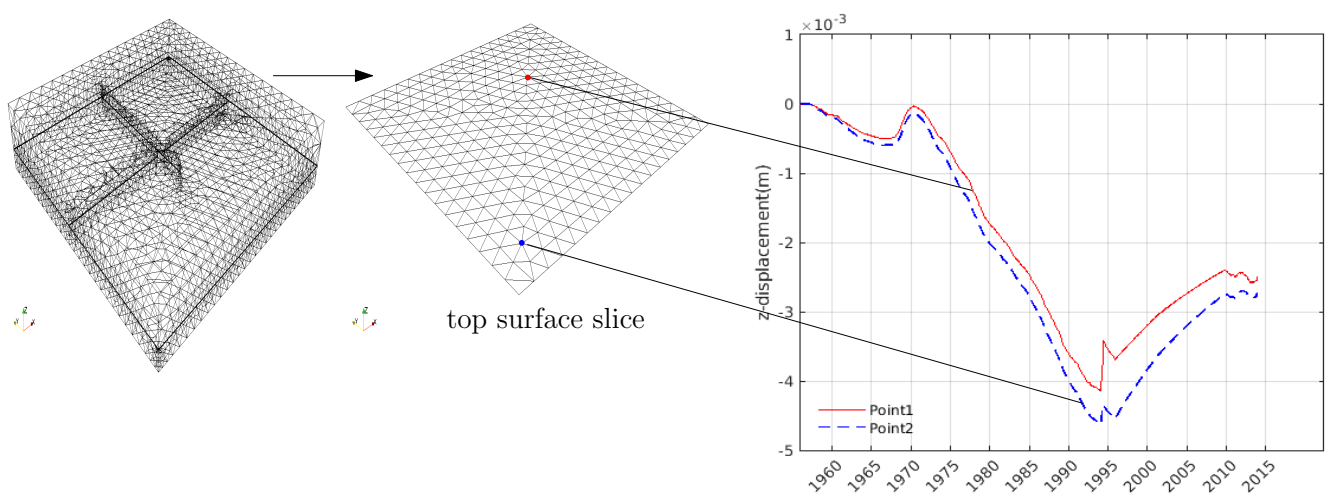

Figure 9: Time series of z-displacement for two nodes on the ground surface. Point 1 (solid line) is located in a fault compartment occupied by many production wells; whereas Point 2 (dash line) is in a larger, hydraulically-connected region with fewer wells. This explains the difference in their displacement magnitudes and trends.

properties or geologic structures. This is difficult to do with a single-grid framework where the grid does not extend to the ground for computational reasons.

\section{Conclusions and outlook}

We presented a computational framework for fast estimation of fault stability and ground deformation in multiphase geomechanics pertaining to a field-scale $\mathrm{CO}_{2}$ storage project in an oilfield. The staggered solution algorithm is augmented with a computational geometry-based feature (Section 3) that allows for the flow and geomechanics sub-problems to be solved on different unstructured tetrahedral grids. Fault stability is estimated by tracking the time series of the change in Coulomb failure function DCFF (Eq. (9)) at critical points on the fault. We observed that the fault stability in the reservoir region is dictated by the pore pressure perturbations while the fault stability in the overburden region is dictated more by the local fault stress field. We also noted from the trends in Fig. 8, that as the $\mathrm{CO}_{2}$ storage is proceeding, the faults will continue to experience a destabilizing effect in the reservoir region. This points to the fact that at a point, the destabilizing effect may increase enough to trigger seismic/aseismic fault slip activity depending on the background stress level and fault's frictional properties. 


\section{Appendix A. Equation of mass conservation for multiphase flow in deformable porous media}

The increment in mean total stress is given by

$$
d \sigma_{v}=\frac{1}{3} \operatorname{tr}(\delta \boldsymbol{\sigma})=\frac{1}{3} \operatorname{tr}\left(\boldsymbol{C}_{d r}: \delta \boldsymbol{\varepsilon}-b \delta \tilde{p} \mathbf{1}\right)=K_{d r} d \varepsilon_{v}-b \delta \tilde{p}
$$

where $K_{d r}$ is drained bulk modulus of porous medium. Eq. (A.1) can also be written as

$$
d \varepsilon_{v}=\frac{d \sigma_{v}}{K_{d r}}+\frac{b d \tilde{p}}{K_{d r}}
$$

From the solid mass conservation equation, the incremental porosity update is given as $[65,66]$

$$
d \phi=\left(\frac{b-\phi}{K_{d r}}\right)\left(d \sigma_{v}+d \tilde{p}\right)
$$

In the deformed configuration, the mass of phase $\alpha$ per unit volume of porous medium is

$$
m_{\alpha}=\rho_{\alpha} S_{\alpha} \phi J
$$

where $J=1+\varepsilon_{v}$ is the Jacobian of deformation under infinitesimal deformation theory. The incremental fluid mass can be written as

$$
\frac{d m_{\alpha}}{\rho_{\alpha}}=\phi J \frac{\partial S_{\alpha}}{\partial P_{\alpha \beta}} d P_{\alpha \beta}+\phi J S_{\alpha} c_{\alpha} d p_{\alpha}+\phi S_{\alpha} d \varepsilon_{v}+J S_{\alpha} d \phi
$$

where $c_{\alpha}=\frac{1}{\rho_{\alpha}} \frac{d \rho_{\alpha}}{d p_{\alpha}}$ is the compressibility of the fluid phase $\alpha, P_{\alpha \beta}=p_{\beta}-p_{\alpha}$ is the capillary pressure and $\partial S_{\alpha} / \partial P_{\alpha \beta}$ is the inverse capillary pressure derivative which can be obtained from one of the $P_{\alpha \beta}$ vs. $S_{\alpha}$ models commonly used in flow simulations, e.g. the van Genuchten model. Substituting Eqs. (A.3) and (A.2) in Eq. (A.4) and assuming $\varepsilon_{v} \ll 1$ and noting that $b_{\alpha}=b S_{\alpha}$, we get

$$
\frac{d m_{\alpha}}{\rho_{\alpha}}=\phi \frac{\partial S_{\alpha}}{\partial P_{\alpha \beta}} d P_{\alpha \beta}+\phi S_{\alpha} c_{\alpha} d p_{\alpha}+(\phi b+b-\phi) \frac{S_{\alpha}}{K_{d r}} d \tilde{p}+\frac{b_{\alpha} d \sigma_{v}}{K_{d r}}
$$


which can be written as

$$
\begin{aligned}
\frac{d m_{\alpha}}{\rho_{\alpha}} & =\phi \frac{\partial S_{\alpha}}{\partial P_{\alpha \beta}} d P_{\alpha \beta}+\phi S_{\alpha} c_{\alpha} d p_{\alpha}+(\phi b+b-\phi) \frac{S_{\alpha}}{K_{d r}} \sum_{\beta} S_{\beta} \delta p_{\beta}+\frac{b_{\alpha} d \sigma_{v}}{K_{d r}} \\
& =\phi \frac{\partial S_{\alpha}}{\partial P_{\alpha \beta}} d P_{\alpha \beta}+\phi S_{\alpha} c_{\alpha} d p_{\alpha}+N S_{\alpha} \sum_{\beta} S_{\beta} \delta p_{\beta}+b^{2} \frac{S_{\alpha}}{K_{d r}} \sum_{\beta} S_{\beta} \delta p_{\beta}+\frac{b_{\alpha} d \sigma_{v}}{K_{d r}}
\end{aligned}
$$

where $N=(b-\phi)(1-b) / K_{d r}$. Defining $N_{\alpha \beta}$ from the first three terms on the right hand side and substituting the above in Eq. (2), we obtain the multiphase flow equation for phase $\alpha$ in a poroelastic medium as:

$$
\frac{\partial}{\partial t}\left(\rho_{\alpha} \sum_{\beta}\left(N_{\alpha \beta}+\frac{b_{\alpha} b_{\beta}}{K_{d r}}\right) p_{\beta}\right)+\frac{1}{K_{d r}} \frac{\partial}{\partial t}\left(\rho_{\alpha} b_{\alpha} \sigma_{v}\right)+\nabla \cdot \boldsymbol{w}_{\alpha}=\rho_{\alpha} f_{\alpha}
$$

\section{Appendix B. Fully discrete system of equations}

Let $\Omega^{p}$ and $\Omega^{f}$ be our domains of interest for the geomechanics and flow problems with closed boundaries $\partial \Omega^{p}$ and $\partial \Omega^{f}$ respectively. The geomechanical equations are solved using the finite element method whereas the multiphase flow equations are solved using the finite volume method.

Appendix B.1. Fully discrete multiphase flow problem

For the two-phase water-gas flow problem, we get

$$
\begin{gathered}
N_{g g}=-\phi \frac{\partial S_{w}}{\partial P_{w g}}+\phi S_{g} c_{g}+S_{g}^{2} N, \\
N_{g w}=N_{w g}=\phi \frac{\partial S_{w}}{\partial P_{w g}}+S_{g} S_{w} N, \\
N_{w w}=-\phi \frac{\partial S_{w}}{\partial P_{w g}}+\phi S_{w} c_{w}+S_{w}^{2} N,
\end{gathered}
$$

Let $\Omega^{f}=\bigcup_{i=1}^{n_{f}} \Omega_{i}$ where $n_{f}$ is the number of flow elements. Note that in each element, $S_{w}+S_{g} \equiv 1$. We integrate the fluid phase mass conservation equation, Eq. (6), for each phase over each element $i$. For the water and gas 
phases, this yields

$$
\begin{aligned}
& \frac{\partial}{\partial t} \int_{\Omega_{i}} \rho_{w}\left(\left(N_{w w}+N_{w g}+\frac{b b_{w}}{K_{d r}}\right) p_{g}-\left(N_{w w}+\frac{b_{w}^{2}}{K_{d r}}\right) P_{w g}\right) d \Omega \\
& +\frac{1}{K_{d r}} \frac{\partial}{\partial t} \int_{\Omega_{i}} \rho_{w} b_{w} \sigma_{v} d \Omega-\int_{\partial \Omega_{i}} \boldsymbol{w}_{w} \cdot \boldsymbol{n}_{i} d \Gamma=\int_{\Omega_{i}} \rho_{w} f_{w} d \Omega \\
& \frac{\partial}{\partial t} \int_{\Omega_{i}} \rho_{g}\left(\left(N_{w g}+N_{g g}+\frac{b b_{g}}{K_{d r}}\right) p_{g}-\left(N_{w g}+\frac{b_{g} b_{w}}{K_{d r}}\right) P_{w g}\right) d \Omega \\
& +\frac{1}{K_{d r}} \frac{\partial}{\partial t} \int_{\Omega_{i}} \rho_{g} b_{g} \sigma_{v} d \Omega-\int_{\partial \Omega_{i}} \boldsymbol{w}_{g} \cdot \boldsymbol{n}_{i} d \Gamma=\int_{\Omega_{i}} \rho_{g} f_{g} d \Omega
\end{aligned}
$$

where we used the capillary pressure relation, $P_{w g}=p_{g}-p_{w}$, to eliminate the water phase pressure, the Biot coefficient relation, $b_{g}+b_{w}=b$, and integration by parts for the mass-flux term to express it as a surface integral. $\boldsymbol{n}_{i}$ is the outward normal to the boundary of element $i$. We approximate both the pressure and the saturation fields with a piecewise constant interpolation function, $\varphi$, such that $\varphi_{i}$ takes a constant value of 1 over element $i$ and 0 at all other elements. Phase pressures and saturations are approximated as

$$
p_{\alpha} \approx p_{\alpha, h}=\sum_{i=1}^{n_{f}} \varphi_{i} p_{\alpha, i}, \quad S_{\alpha} \approx S_{\alpha, h}=\sum_{i=1}^{n_{f}} \varphi_{i} S_{\alpha, i}
$$

where the discrete pressures, $p_{\alpha, i}$, and phase saturations, $S_{\alpha, i}$, are located at the center of element $i$. We can further express the mass flux term as a sum of integral fluxes between element $i$ and its adjacent elements $j$ :

$$
\int_{\partial \Omega_{i}} \boldsymbol{w}_{w} \cdot \boldsymbol{n}_{i} d \Gamma=\sum_{j=1}^{n_{\text {face }, i}} \int_{\Gamma_{i j}} \boldsymbol{w}_{w} \cdot \boldsymbol{n}_{i j} d \Gamma=\sum_{j=1}^{n_{\text {face }, i}} W_{w, i j}
$$

where $n_{\text {face }, i}$ is the number of faces of element $i$ ( $n_{\mathrm{face}, i}=4$ for a tetrahedral mesh such as the ones we used for the Mandel and Farnsworth models above and $n_{\text {face }, i}=6$ for a hexahedral mesh), and $\boldsymbol{n}_{i j}$ is the outward normal at the face $\Gamma_{i j}$. The inter-element flux $W_{w, i j}$, is evaluated using a two-point flux approximation method which uses the flow potentials, $\Phi_{w, i}=p_{w, i}-$ $\rho_{w, i} g z_{i}$ and $\Phi_{w, j}=p_{w, j}-\rho_{w, j} g z_{j}$, to approximate the flux through interface $i j$ between two neighboring elements calculated from upstream cells as follows:

$$
W_{w, i j}= \begin{cases}A_{i j} /\left(\ell_{i} / k_{i}+\ell_{j} / k_{j}\right) \rho_{w, i} k_{w, i}^{r} / \mu_{w, i}\left(\Phi_{w, i}-\Phi_{w, j}\right), & \text { if } \Phi_{w, i}>\Phi_{w, j}, \\ A_{i j} /\left(\ell_{i} / k_{i}+\ell_{j} / k_{j}\right) \rho_{w, j} k_{w, j}^{r} / \mu_{w, j}\left(\Phi_{w, i}-\Phi_{w, j}\right), & \text { otherwise }\end{cases}
$$


where $z_{i}$ and $z_{j}$ are the centroid depths of the elements (with $z$-axis pointing downward), $A_{i j}$ is the area of the interface, $\ell_{i}$ and $\ell_{j}$ are the distances between the centroid of the interface and the centroid of the respective adjacent elements, and $k_{i}$ and $k_{j}$ are the element permeabilities. Using the notation $\delta_{t}(\cdot) \equiv \frac{(\cdot)^{n+1}-(\cdot)^{n}}{\Delta t}$, the fully discrete system of equations is

$$
\begin{aligned}
R_{g, i}^{n+1} & =\delta_{t}\left[V_{b} \rho_{g}\left(\left(N_{g w}+N_{g g}+\frac{b b_{g}}{K_{d r}}\right) p_{g}-\left(N_{g w}+\frac{b_{g} b_{w}}{K_{d r}}\right) P_{w g}\right)\right]_{i} \\
& +\delta_{t}\left[\rho_{g} \frac{b_{g}}{K_{d r}} \sigma_{v} V_{b}\right]_{i}-\sum_{j=1}^{n_{\text {face }, i}} W_{g, i j}^{n+1}-\left[\rho_{g}^{n+1} f_{g}^{n+1} V_{b}\right]_{i} \\
R_{w, i}^{n+1} & =\delta_{t}\left[V_{b} \rho_{w}\left(\left(N_{w w}+N_{w g}+\frac{b b_{w}}{K_{d r}}\right) p_{g}-\left(N_{w w}+\frac{b_{w}^{2}}{K_{d r}}\right) P_{w g}\right)\right]_{i} \\
& +\delta_{t}\left[\rho_{w} \frac{b_{w}}{K_{d r}} \sigma_{v} V_{b}\right]_{i}-\sum_{j=1}^{n_{\text {face }, i}} W_{w, i j}^{n+1}-\left[\rho_{w}^{n+1} f_{w}^{n+1} V_{b}\right]_{i}
\end{aligned}
$$

where subscript $i \in\left[1, n_{f}\right]$ refers to the value at element $i$. It is important to note that $\delta_{t} \sigma_{v}$ is the same across all Newton iterations within a fixed stress iteration in lieu of the fixed stress constraint. The global residual vectors are obtained by assembly of the element residual vectors: $\boldsymbol{R}_{g}=$ $\left[R_{g, 1}, \ldots, R_{g, n_{f}}\right]^{T}, \boldsymbol{R}_{w}=\left[R_{w, 1}, \ldots, R_{w, n_{f}}\right]^{T}$. In vector form, the system of $n_{\text {phase }} \times n_{f}$ algebraic equations of the flow problem reads:

$$
\left[\begin{array}{l}
\boldsymbol{R}_{g} \\
\boldsymbol{R}_{w}
\end{array}\right]^{n+1}=\left[\begin{array}{l}
\mathbf{0} \\
\mathbf{0}
\end{array}\right]
$$

which must be solved at every time step for the vector of element gas-phase pressures and water-phase saturations

$$
\boldsymbol{P}_{g}^{n+1}=\left[p_{g, 1}^{n+1}, \ldots, p_{g, n_{f}}^{n+1}\right]^{T}, \quad \boldsymbol{S}_{w}^{n+1}=\left[S_{w, 1}^{n+1}, \ldots, S_{w, n_{f}}^{n+1}\right]^{T}
$$

We solve the linear system of equations of the multiphase flow problem using Newton's method. The correction vector is the solution of the linear system of equations:

$$
\left[\begin{array}{ll}
\frac{\partial \boldsymbol{R}_{g}}{\partial \boldsymbol{P}_{g}} & \frac{\partial \boldsymbol{R}_{g}}{\partial \boldsymbol{S}_{w}} \\
\frac{\partial \boldsymbol{R}_{w}}{\partial \boldsymbol{P}_{g}} & \frac{\partial \boldsymbol{R}_{w}}{\partial \boldsymbol{S}_{w}}
\end{array}\right]^{(k)}\left[\begin{array}{l}
\delta \boldsymbol{P}_{g} \\
\delta \boldsymbol{S}_{w}
\end{array}\right]^{(k)}=-\left[\begin{array}{c}
\boldsymbol{R}_{g} \\
\boldsymbol{R}_{w}
\end{array}\right]^{(k)},
$$

where the partial derivatives of the residuals are evaluated using Eq. (B.1). 


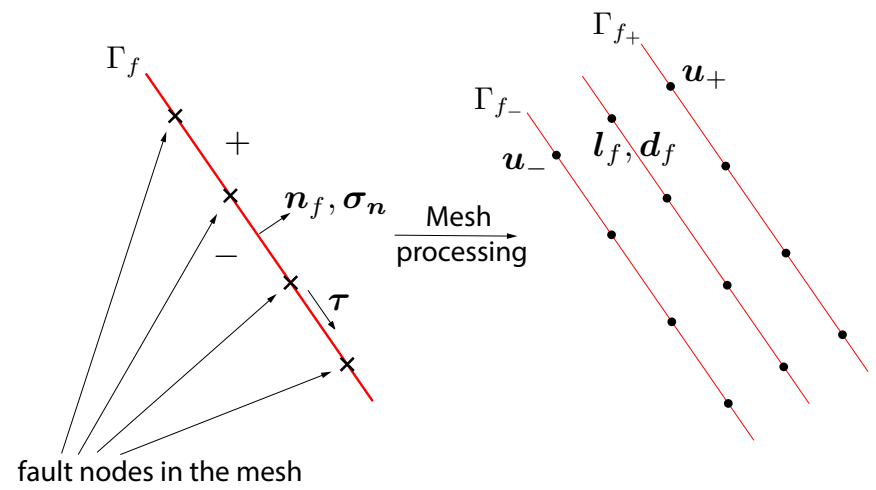

Figure B.10: A fault surface $\Gamma_{f}$ (shown as a line in a $2 \mathrm{D}$ domain) is processed during mesh processing to create three surfaces: the positive side surface $\Gamma_{f+}$ containing $\boldsymbol{u}_{+}$, the negative side surface $\Gamma_{f-}$ containing $\boldsymbol{u}_{-}$, and the middle slip surface containing the fault effective traction vector $\boldsymbol{l}_{f}$ and the slip vector $\boldsymbol{d}_{f}$. The fault surface need not be planar i.e. it can be curved with the normal vector $\boldsymbol{n}_{f}$ varying in space on the fault surface.

\section{Appendix B.2. Fully discrete geomechanics problem}

We treat faults as surfaces of discontinuity embedded in the continuum across which displacement is allowed to be discontinuous to recognize the possibility of fault slip As shown in Fig. B.10, each node on the fault is triplicated to create a "+" node, a "-" node, and a Lagrange node in the middle. The side nodes store the positive side displacement $\boldsymbol{u}_{+}$and the negative side displacement $\boldsymbol{u}_{-}$, respectively, of the adjacent rock hosting the fault. All three nodes are physically collocated on the initial grid, so the cohesive elements representing the fault are zero thickness elements at $t=0$ before any fault slip or opening. Slip on the fault is the displacement of the positive side relative to the negative side,

$$
\left(\boldsymbol{u}_{+}-\boldsymbol{u}_{-}\right)-\boldsymbol{d}_{f}=\mathbf{0} \text { on } \Gamma_{f},
$$

where $\boldsymbol{d}_{f}$ is the fault slip vector. We impose the effective traction on the fault by introducing a Lagrange multiplier, $\boldsymbol{l}_{f}$, which is a force per unit area required to satisfy Eq. B.7. The Lagrange node stores the Lagrange multiplier $\boldsymbol{l}_{f}$ and the fault slip $\boldsymbol{d}_{f}$. The problem statement is to find $\left(\boldsymbol{u}, \boldsymbol{l}_{f}\right)$ belonging to appropriate functional spaces satisfying the essential boundary conditions 
$\left(\boldsymbol{u}=\overline{\boldsymbol{u}}\right.$ on $\left.\Gamma_{u}\right)$ such that

$$
\begin{aligned}
& \int_{\Omega^{p}} \nabla^{s} \boldsymbol{\eta}:\left(\boldsymbol{\sigma}^{\prime}-b \tilde{p} \mathbf{1}\right) d \Omega^{p}+\int_{\Gamma_{f_{+}}} \boldsymbol{\eta} \cdot\left(\boldsymbol{l}_{f}-b p_{f} \boldsymbol{n}\right) d \Gamma \\
& -\int_{\Gamma_{f_{-}}} \boldsymbol{\eta} \cdot\left(\boldsymbol{l}_{f}-b p_{f} \boldsymbol{n}\right) d \Gamma-\int_{\Omega^{p}} \boldsymbol{\eta} \cdot \rho_{b} \boldsymbol{g} d \Omega^{p}-\int_{\Gamma_{\sigma}} \boldsymbol{\eta} \cdot \overline{\boldsymbol{t}} d \Gamma=0, \\
& \int_{\Gamma_{f_{+}}} \boldsymbol{\eta} \cdot \boldsymbol{u}_{+} d \Gamma-\int_{\Gamma_{f_{-}}} \boldsymbol{\eta} \cdot \boldsymbol{u}_{-} d \Gamma-\int_{\Gamma_{f}} \boldsymbol{\eta} \cdot \boldsymbol{d}_{f} d \Gamma=0,
\end{aligned}
$$

for all test functions $\boldsymbol{\eta}\left(n_{\operatorname{dim}} \times 1\right.$ vector $)$ belonging to the appropriate functional space satisfying $\boldsymbol{\eta}=\mathbf{0}$ on $\Gamma_{u}$. $\overline{\boldsymbol{t}}$ is the prescribed traction on the traction boundary $\Gamma_{\sigma}$ of the domain, e.g. $\overline{\boldsymbol{t}}=\mathbf{0}$ on the traction-free ground surface. Here, we used the multiphase effective stress equation (Eq. (3)), $p_{f}$ is the fault pressure, and $\rho_{b}$ is the bulk density, all of which depend on the phase pressures and saturations, and, therefore, on the solution of the flow problem. Let the domain be partitioned into non-overlapping elements (grid blocks), $\Omega^{p}=\bigcup_{j=1}^{n_{\text {elem }}} \Omega_{j}$ where $n_{\text {elem }}$ is the number of geomechanics elements. For the remainder of this section, we shall use the variable $\Omega$ instead of $\Omega^{p}$ for the sake of notational convenience. A fault is treated as an interior boundary with its domain, $\Gamma_{f}$, partitioned into non-overlapping fault elements, $\Gamma_{f}=\bigcup_{j=1}^{n_{f, \text { elem }}} \Gamma_{f, j}$, where the subscript $f$ indicates variables associated with a fault. The displacement, the Lagrange multiplier, and the slip fields are approximated as follows:

$$
\boldsymbol{u} \approx \boldsymbol{u}_{h}=\sum_{b=1}^{n_{\text {node }}} \eta_{b} \boldsymbol{U}_{b}, \quad \boldsymbol{l}_{f} \approx \boldsymbol{l}_{h}=\sum_{\bar{b}=1}^{n_{f, \text { node }}} \eta_{\bar{b}} \boldsymbol{L}_{\bar{b}}, \quad \boldsymbol{d}_{f} \approx \boldsymbol{d}_{h}=\sum_{\bar{b}=1}^{n_{f, \text { node }}} \eta_{\bar{b}} \boldsymbol{D}_{\bar{b}},
$$

where subscript $h$ indicates the finite element approximation, $n_{\text {node }}$ is the number of displacement nodes (including the positive and negative side nodes at the fault elements, and $n_{f \text {,node }}$ is the number of Lagrange nodes. $\boldsymbol{U}_{b}$ is the $n_{\text {dim }} \times 1$ vector of nodal displacements for node $b$ in the domain coordinate system. $\boldsymbol{L}_{\bar{b}}$ and $\boldsymbol{D}_{\bar{b}}$ are the $n_{\text {dim }} \times 1$ Lagrange multiplier and slip vectors at Lagrange node $\bar{b}$ of the fault, and they are in the fault coordinate system defined by left-lateral, reverse, and opening directions. After substitution of the finite element approximations into the weak form of the problem (Eqs. (B.7)(B.8)), we obtain the discrete equations in residual form at all displacement 
nodes $a$ and all Lagrange nodes $\bar{a}$ :

$$
\begin{aligned}
\mathbf{0} & =\int_{\Omega} \boldsymbol{B}_{a}^{T}\left(\boldsymbol{\sigma}_{h}^{\prime}-b \tilde{p}_{h} \mathbf{1}\right) d \Omega+\int_{\Gamma_{f_{+}}} \boldsymbol{\eta}_{a}^{T}\left(\boldsymbol{l}_{h}-b p_{f, h} \boldsymbol{n}\right) d \Gamma \\
& -\int_{\Gamma_{f_{-}}} \boldsymbol{\eta}_{a}^{T}\left(\boldsymbol{l}_{h}-b p_{f, h} \boldsymbol{n}\right) d \Gamma-\int_{\Omega} \boldsymbol{\eta}_{a}^{T} \rho_{b, h} \boldsymbol{g} d \Omega-\int_{\Gamma_{\sigma}} \boldsymbol{\eta}_{a}^{T} \overline{\boldsymbol{t}} d \Gamma, \\
\mathbf{0} & =\int_{\Gamma_{f_{+}}} \boldsymbol{\eta}_{\bar{a}}^{T} \boldsymbol{u}_{h_{+}} d \Gamma-\int_{\Gamma_{f_{-}}} \boldsymbol{\eta}_{\bar{a}}^{T} \boldsymbol{u}_{h_{-}} d \Gamma-\int_{\Gamma_{f}} \boldsymbol{\eta}_{\bar{a}}^{T} \boldsymbol{d}_{h} d \Gamma
\end{aligned}
$$

where $\boldsymbol{\eta}_{a}$ and $\boldsymbol{B}_{a}$ are nodal matrices for the shape function and its gradient respectively. Equation (B.9) results in $n_{\operatorname{dim}} \times n_{\text {node }}$ equations, and Eq. (B.10) results in $n_{\text {dim }} n_{f, \text { node }}$ equations. Since we use the prescribed fault rupture model, $\delta \boldsymbol{D}_{\bar{a}}$ is prescribed at all fault nodes. The fully discrete system of equations is

$$
\begin{aligned}
\boldsymbol{R}_{u, a}^{n+1} & =\int_{\Omega} \boldsymbol{B}_{a}^{T}\left(\boldsymbol{\sigma}_{h}^{\prime n+1}-b \tilde{p}_{h}^{n+1} \mathbf{1}\right) d \Omega+\int_{\Gamma_{f_{+}}} \boldsymbol{\eta}_{a}^{T}\left(\boldsymbol{l}_{h}^{n+1}-b p_{f, h}^{n+1} \boldsymbol{n}\right) d \Gamma \\
& -\int_{\Gamma_{f_{-}}} \boldsymbol{\eta}_{a}^{T}\left(\boldsymbol{l}_{h}^{n+1}-b p_{f, h}^{n+1} \boldsymbol{n}\right) d \Gamma-\int_{\Omega} \boldsymbol{\eta}_{a}^{T} \rho_{b, h}^{n+1} \boldsymbol{g} d \Omega-\int_{\Gamma_{\sigma}} \boldsymbol{\eta}_{a}^{T} \overline{\boldsymbol{t}} d \Gamma=\mathbf{0} \\
\boldsymbol{R}_{l, \bar{a}}^{n+1} & =\int_{\Gamma_{f_{+}}} \boldsymbol{\eta}_{\bar{a}}^{T} \boldsymbol{u}_{h_{+}}^{n+1} d \Gamma-\int_{\Gamma_{f_{-}}} \boldsymbol{\eta}_{\bar{a}}^{T} \boldsymbol{u}_{h_{-}}^{n+1} d \Gamma-\int_{\Gamma_{f}} \boldsymbol{\eta}_{\bar{a}}^{T} \boldsymbol{d}_{h}^{n+1} d \Gamma=\mathbf{0}
\end{aligned}
$$

The global residual vectors are obtained by assembly of the nodal residual vectors: $\boldsymbol{R}_{u}=\left[\boldsymbol{R}_{u, 1}, \ldots, \boldsymbol{R}_{u, n_{\text {node }}}\right]^{T}, \boldsymbol{R}_{l}=\left[\boldsymbol{R}_{l, 1}, \ldots, \boldsymbol{R}_{l, n_{\mathrm{f}, \text { node }}}\right]^{T}$. In vector form, the system of $\left(n_{\operatorname{dim}} n_{\text {node }}+n_{\operatorname{dim}} n_{f, \text { node }}\right)$ algebraic equations of the mechanical problem reads:

$$
\left[\begin{array}{l}
\boldsymbol{R}_{u} \\
\boldsymbol{R}_{l}
\end{array}\right]^{n+1}=\left[\begin{array}{l}
\mathbf{0} \\
\mathbf{0}
\end{array}\right],
$$

which needs to be solved for the displacements $\boldsymbol{U}^{n+1}=\left[\boldsymbol{U}_{1}^{n+1}, \ldots, \boldsymbol{U}_{n_{\text {node }}}^{n+1}\right]^{T}$ and the Lagrange multipliers $\boldsymbol{L}^{n+1}=\left[\boldsymbol{L}_{1}^{n+1}, \ldots, \boldsymbol{L}_{n_{\mathrm{f}, \text { node }}}^{n+1}\right]^{T}$, subject to the constraint Eq. (B.6) at every time step. We solve the linear systems of equations of the mechanical problem using Newton's method. Given an approximation $\left[\boldsymbol{U}^{n+1}, \boldsymbol{L}^{n+1}\right]^{(k)}$ to the solution at $t_{n+1}$, an improved solution is obtained as

$$
\left[\boldsymbol{U}^{n+1}, \boldsymbol{L}^{n+1}\right]^{(k+1)}=\left[\boldsymbol{U}^{n+1}, \boldsymbol{L}^{n+1}\right]^{(k)}+\left[\delta \boldsymbol{U}^{n+1}, \delta \boldsymbol{L}^{n+1}\right]^{(k)}
$$


where the correction vector is the solution to the system of linear equations (removing the explicit reference to the time level $n+1$ ):

$$
\left[\begin{array}{cc}
\boldsymbol{K} & \boldsymbol{C}^{T} \\
\boldsymbol{C} & \mathbf{0}
\end{array}\right]^{(k)}\left[\begin{array}{c}
\delta \boldsymbol{U} \\
\delta \boldsymbol{L}
\end{array}\right]^{(k)}=-\left[\begin{array}{c}
\boldsymbol{R}_{u} \\
\boldsymbol{R}_{l}
\end{array}\right]^{(k)},
$$

where the block matrices are obtained via element-by-element assembly of the individual nodal contributions to the Jacobian:

$$
\begin{aligned}
\boldsymbol{K}_{a b} & =\frac{\partial \boldsymbol{R}_{u, a}}{\partial \boldsymbol{U}_{b}}=\int_{\Omega} \boldsymbol{B}_{a}^{T} \mathbf{D} \boldsymbol{B}_{b} d \Omega \\
\boldsymbol{C}_{a \bar{b}}^{T} & =\frac{\partial \boldsymbol{R}_{u, a}}{\partial \boldsymbol{L}_{\bar{b}}}=\int_{\Gamma_{f_{+}}} \boldsymbol{\eta}_{a}^{T} \boldsymbol{\eta}_{\bar{b}} d \Gamma-\int_{\Gamma_{f_{-}}} \boldsymbol{\eta}_{a}^{T} \boldsymbol{\eta}_{\bar{b}} d \Gamma
\end{aligned}
$$

Matrix $\boldsymbol{C}$ is the part of the Jacobian associated with the slip constraint, Eq. (B.10), and consists of direction cosine matrices to convert from the global coordinate system to the fault coordinate system (vice versa for $\boldsymbol{C}^{T}$ ). Note that for a linear elastic material with time-independent material properties and boundary conditions, the Jacobian matrix does not change with time, although the residuals may change due to coupling with the flow and due to fault slip. This is a saddle-point problem.

\section{References}

[1] T. Rodosta, W. Aljoe, G. Bromhal, D. Damiani, U.s. doe regional carbon sequestration partnership initiative: New insights and lessons learned, Energy Procedia 114 (2017) 5580-5592.

[2] R. Balch, B. McPherson, et al., Integrating enhanced oil recovery and carbon capture and storage projects: a case study at farnsworth field, texas, in: SPE Western Regional Meeting, Society of Petroleum Engineers, 2016.

[3] N. R. Council, et al., Induced seismicity potential in energy technologies, National Academies Press, 2013.

[4] M. D. Zoback, S. M. Gorelick, Earthquake triggering and large-scale geologic storage of carbon dioxide, Proc. Natl. Acad. Sci. U.S.A. 109 (2012) 10164-10168. 
[5] M. L. Szulczewski, C. W. MacMinn, H. J. Herzog, R. Juanes, Lifetime of carbon capture and storage as a climate-change mitigation technology, Proc. Natl. Acad. Sci. U.S.A. 109 (2012) 5185-5189.

[6] R. Juanes, B. H. Hager, H. J. Herzog, No geologic evidence that seismicity causes fault leakage that would render large-scale carbon capture and storage unsuccessful, Proc. Natl. Acad. Sci. U.S.A. 109 (2012) E3623.

[7] M. D. Zoback, S. M. Gorelick, Reply to Juanes et al.: Evidence that earthquake triggering could render long-term carbon storage unsuccessful in many regions, Proc. Natl. Acad. Sci. U.S.A. 109 (2012) E3624.

[8] M. Preisig, J. H. Prévost, Coupled multi-phase thermo-poromechanical effects. case study: Co2 injection at in salah, algeria, International Journal of Greenhouse Gas Control 5 (2011) 1055-1064.

[9] J. Rutqvist, J. Birkholzer, F. Cappa, C.-F. Tsang, Estimating maximum sustainable injection pressure during geological sequestration of co2 using coupled fluid flow and geomechanical fault-slip analysis, Energy Conversion and Management 48 (2007) 1798-1807.

[10] J. Rutqvist, J. Birkholzer, C.-F. Tsang, Coupled reservoirgeomechanical analysis of the potential for tensile and shear failure associated with co2 injection in multilayered reservoir-caprock systems, International Journal of Rock Mechanics and Mining Sciences 45 (2008) $132-143$.

[11] J. E. Streit, R. R. Hillis, Estimating fault stability and sustainable fluid pressures for underground storage of co2 in porous rock, Energy 29 (2004) 1445-1456.

[12] P. Teatini, N. Castelletto, G. Gambolati, 3d geomechanical modeling for co2 geological storage in faulted formations. a case study in an offshore northern adriatic reservoir, italy, International Journal of Greenhouse Gas Control 22 (2014) 63-76.

[13] J. Verdon, J.-M. Kendall, D. White, D. Angus, Linking microseismic event observations with geomechanical models to minimise the risks of storing co2 in geological formations, Earth and Planetary Science Letters 305 (2011) 143-152. 
[14] V. Vilarrasa, J. Carrera, S. Olivella, J. Rutqvist, L. Laloui, Induced seismicity in geologic carbon storage, Solid Earth 10 (2019) 871-892.

[15] J. J. Bommer, S. Oates, J. M. Cepeda, C. Lindholm, J. Bird, R. Torres, G. Marroquín, J. Rivas, Control of hazard due to seismicity induced by a hot fractured rock geothermal project, Engineering Geology 83 (2006) 287-306. doi:https://doi.org/10.1016/j.enggeo.2005.11.002.

[16] S. Dana, B. Ganis, M. F. Wheeler, A multiscale fixed stress split iterative scheme for coupled flow and poromechanics in deep subsurface reservoirs, Journal of Computational Physics 352 (2018) 1-22.

[17] J. Kim, H. Tchelepi, R. Juanes, Stability, accuracy and efficiency of sequential methods for coupled flow and geomechanics, SPE Journal 16 (2011) 249-262.

[18] A. Mikelić, M. Wheeler, Convergence of iterative coupling for coupled flow and geomechanics, Computational Geosciences 17 (2013) 455-461.

[19] A. Mikelić, B. Wang, M. F. Wheeler, Numerical convergence study of iterative coupling for coupled flow and geomechanics, Computational Geosciences 18 (2014) 325-341.

[20] N. Castelletto, J. A. White, H. A. Tchelepi, Accuracy and convergence properties of the fixed-stress iterative solution of two-way coupled poromechanics, International Journal for Numerical and Analytical Methods in Geomechanics 39 (2015) 1593-1618.

[21] J. A. White, N. Castelletto, H. A. Tchelepi, Block-partitioned solvers for coupled poromechanics: A unified framework, Computer Methods in Applied Mechanics and Engineering 303 (2016) 55-74.

[22] N. Castelletto, J. A. White, M. Ferronato, Scalable algorithms for threefield mixed finite element coupled poromechanics, Journal of Computational Physics 327 (2016) 894-918.

[23] T. Almani, K. Kumar, A. Dogru, G. Singh, M. F. Wheeler, Convergence analysis of multirate fixed-stress split iterative schemes for coupling flow with geomechanics, Computer Methods in Applied Mechanics and Engineering 311 (2016) 180-207. 
[24] T. Almani, K. Kumar, M. F. Wheeler, Convergence and error analysis of fully discrete iterative coupling schemes for coupling flow with geomechanics, Computational Geosciences (2017).

[25] J. W. Both, K. Kumar, J. M. Nordbotten, F. A. Radu, Anderson accelerated fixed-stress splitting schemes for consolidation of unsaturated porous media, Computers and Mathematics with Applications 77 (2019) 1479 - 1502. 7th International Conference on Advanced Computational Methods in Engineering (ACOMEN 2017).

[26] J. A. White, N. Castelletto, S. Klevtsov, Q. M. Bui, D. Osei-Kuffuor, H. A. Tchelepi, A two-stage preconditioner for multiphase poromechanics in reservoir simulation, Computer Methods in Applied Mechanics and Engineering 357 (2019) 112575.

[27] M. Borregales, K. Kumar, F. A. Radu, C. Rodrigo, F. J. Gaspar, A partially parallel-in-time fixed-stress splitting method for biot's consolidation model, Computers and Mathematics with Applications 77 (2019) 1466 - 1478. 7th International Conference on Advanced Computational Methods in Engineering (ACOMEN 2017).

[28] E. Storvik, J. W. Both, K. Kumar, J. M. Nordbotten, F. A. Radu, On the optimization of the fixed-stress splitting for biot's equations, International Journal for Numerical Methods in Engineering (2019) nme.6130.

[29] F. A. Radu, J. M. Nordbotten, I. S. Pop, K. Kumar, A robust linearization scheme for finite volume based discretizations for simulation of two-phase flow in porous media, Journal of Computational and Applied Mathematics 289 (2015) $134-141$.

[30] E. Ahmed, J. M. Nordbotten, F. A. Radu, Adaptive asynchronous timestepping, stopping criteria, and a posteriori error estimates for fixedstress iterative schemes for coupled poromechanics problems, Journal of Computational and Applied Mathematics 364 (2020) 112312.

[31] X. Lu, M. F. Wheeler, Three-way coupling of multiphase flow and poromechanics in porous media, Journal of Computational Physics 401 (2020) 109053. 
[32] T. Kadeethum, S. Lee, F. Ballarin, J. Choo, H. Nick, A locally conservative mixed finite element framework for coupled hydro-mechanicalchemical processes in heterogeneous porous media, arXiv preprint arXiv:2010.04994 (2020).

[33] Y. Liu, L. Liu, J. Y. Leung, K. Wu, G. Moridis, Coupled flow/geomechanics modeling of interfracture water injection to enhance oil recovery in tight reservoirs, SPE Journal 26 (2021) 1-21.

[34] Y. Liu, L. Liu, J. Y. Leung, G. J. Moridis, Sequentially coupled flow and geomechanical simulation with a discrete fracture model for analyzing fracturing fluid recovery and distribution in fractured ultra-low permeability gas reservoirs, Journal of Petroleum Science and Engineering 189 (2020) 107042.

[35] L. Liu, Y. Liu, J. Yao, Z. Huang, et al., Efficient coupled multiphase-flow and geomechanics modeling of well performance and stress evolution in shale-gas reservoirs considering dynamic fracture properties, SPE Journal (2020).

[36] O. Duran, M. Sanei, P. R. Devloo, E. S. Santos, An enhanced sequential fully implicit scheme for reservoir geomechanics, Computational Geosciences 24 (2020) 1557-1587.

[37] S. B. Bosma, S. Klevtsov, O. Møyner, N. Castelletto, Enhanced multiscale restriction-smoothed basis (msrsb) preconditioning with applications to porous media flow and geomechanics, Journal of Computational Physics 428 (2021) 109934.

[38] S. Dana, M. F. Wheeler, Convergence analysis of fixed stress split iterative scheme for small strain anisotropic poroelastoplasticity: a primer, arXiv preprint arXiv:1810.04163 (2018).

[39] S. Dana, M. F. Wheeler, The correspondence between voigt and reuss bounds and the decoupling constraint in a two-grid staggered solution algorithm to coupled flow and deformation in heterogeneous poroelastic media, arXiv preprint arXiv:1810.09443 (2018).

[40] S. Dana, M. F. Wheeler, Augmented lagrangian for treatment of hanging nodes in hexahedral meshes, arXiv preprint arXiv:1809.04031 (2018). 
[41] S. Dana, K. R. Lyathakula, Uncertainty quantification in friction model for earthquakes using bayesian inference, arXiv preprint arXiv:2104.11156 (2021).

[42] S. Dana, M. F. Wheeler, Convergence analysis of fixed stress split iterative scheme for anisotropic poroelasticity with tensor biot parameter, Computational Geosciences 22 (2018) 1219-1230.

[43] S. Dana, M. F. Wheeler, Convergence analysis of two-grid fixed stress split iterative scheme for coupled flow and deformation in heterogeneous poroelastic media, Computer Methods in Applied Mechanics and Engineering 341 (2018) 788-806.

[44] S. Dana, M. F. Wheeler, Design of convergence criterion for fixed stress split iterative scheme for small strain anisotropic poroelastoplasticity coupled with single phase flow, arXiv preprint arXiv:1912.06476 (2019).

[45] S. Dana, M. F. Wheeler, An efficient algorithm for numerical homogenization of fluid filled porous solids: part-i, arXiv preprint arXiv:2002.03770 (2020).

[46] S. Dana, M. F. Wheeler, A machine learning accelerated $\mathrm{fe}^{2}$ homogenization algorithm for elastic solids, arXiv preprint arXiv:2003.11372 (2020).

[47] S. Dana, S. Srinivasan, S. Karra, N. Makedonska, J. D. Hyman, D. O'Malley, H. Viswanathan, G. Srinivasan, Towards real-time forecasting of natural gas production by harnessing graph theory for stochastic discrete fracture networks, Journal of Petroleum Science and Engineering 195 (2020) 107791.

[48] S. Dana, M. Jammoul, M. F. Wheeler, Performance studies of the fixed stress split algorithm for immiscible two-phase flow coupled with linear poromechanics, Computational Geosciences (2021) 1-15.

[49] S. Dana, J. Ita, M. F. Wheeler, The correspondence between voigt and reuss bounds and the decoupling constraint in a two-grid staggered algorithm for consolidation in heterogeneous porous media, Multiscale Modeling \& Simulation 18 (2020) 221-239. 
[50] S. Dana, A simple framework for arriving at bounds on effective moduli in heterogeneous anisotropic poroelastic solids, arXiv preprint arXiv:1912.10835 (2019).

[51] S. Dana, System of equations and staggered solution algorithm for immiscible two-phase flow coupled with linear poromechanics, arXiv preprint arXiv:1912.04703 (2019).

[52] S. Dana, Addressing challenges in modeling of coupled flow and poromechanics in deep subsurface reservoirs, Ph.D. thesis, The University of Texas at Austin, 2018.

[53] O. Coussy, Mechanics of Porous Continua, John Wiley and Sons, Chichester, England, 1995.

[54] M. Muskat, Physical Principles of Oil Production, McGraw-Hill, New York, 1949.

[55] J. Bear, Dynamics of Fluids in Porous Media, Wiley, New York, 1972.

[56] M. H. Austern, Generic programming and the STL: using and extending the $\mathrm{C}++$ Standard Template Library, Addison-Wesley Longman Publishing Co., Inc., 1998.

[57] M. D. White, R. Esser, B. McPherson, R. Balch, N. Liu, P. Rose, L. Garcia, W. Ampomah, Interpretation of tracer experiments on inverted five-spot well-patterns within the western half of the farnsworth unit oil field, Energy Procedia 114 (2017) 7070-7095.

[58] A. C. Hutton, Geophysical modeling and structural interpretation of a 3D reflection seismic survey in Farnsworth Unit, TX, New Mexico Institute of Mining and Technology, 2015.

[59] R. Balch, R. Will*, G. El-Kaseeh, R. Grigg, A. Hutton, P. Czoski, Integrating multi-scale seismic measurements for eor/ccus, in: SEG Technical Program Expanded Abstracts 2015, Society of Exploration Geophysicists, 2015, pp. 2837-2841.

[60] S. R. Gallagher, Depositional and diagenetic controls on reservoir heterogeneity: upper morrow sandstone, Farnsworth unit, Ochiltree county, Texas, Ph.D. thesis, New Mexico Tech, 2014. 
[61] D. Rose-Coss, W. Ampomah, A. C. Hutton, E. Gragg, P. Mozley, R. S. Balch, R. Grigg, Geologic characterization for co 2-eor simulation: A case study of the farnsworth unit, anadarko basin, texas, in: AAPG Annual Convention and Exhibition, 2015.

[62] W. Ampomah, R. Balch, D. Ross-Coss, A. Hutton, M. Cather, R. Will, et al., An integrated approach for characterizing a sandstone reservoir in the anadarko basin, in: Offshore Technology Conference, Offshore Technology Conference, 2016.

[63] G. El-kaseeh, R. Will, R. Balch, R. Grigg, Multi-scale seismic measurements for co2 monitoring in an eor/ccus project, Energy Procedia 114 (2017) 3656-3670.

[64] K. Aziz, A. Settari, Petroleum Reservoir Simulation, Elsevier, London, 1979.

[65] J. Geertsma, The effect of fluid pressure decline on volumetric changes of porous rocks, SPE 210 (1957) 331-340.

[66] R. J. S. Brown, J. Korringa, On the dependence of the elastic properties of a porous rock on the compressibility of the pore fluid, Geophysics 40 (1975) 608-616. 